\title{
Space and observers in cosmology
}

\author{
M. Lachièze-Rey ${ }^{\star}$ \\ Service d'Astrophysique, CE Saclay, 91191 Gif-sur-Yvette Cedex, France \\ Received 16 May 2001 / Accepted 29 June 2001

\begin{abstract}
I provide a prescription to define space, at a given moment, for an arbitrary observer in an arbitrary (sufficiently regular) curved space-time. This prescription, based on synchronicity (simultaneity) arguments, defines a foliation of space-time, which corresponds to a family of canonically associated observers. It provides also a natural global reference frame (with space and time coordinates) for the observer, in space-time (or rather in the part of it which is causally connected to him), which remains Minkowskian along his world-line. This definition intends to provide a basis for the problem of quantization in curved space-time, and/or for non inertial observers. Application to Minkowski spacetime illustrates clearly the fact that different observers see different spaces. For example, it allows one to define space everywhere without ambiguity, for the Langevin observer (involved in the Langevin pseudoparadox of twins). Applied to the Rindler observer (with uniform acceleration) it leads to the Rindler coordinates, whose choice is so justified with a physical basis. This leads to an interpretation of the Unruh effect, as due to the observer-dependence of the definition of space (and time). This prescription is also applied in cosmology, for inertial observers in the Friedmann-Lemaître models: space for the observer appears to differ from the hypersurfaces of homogeneity, which do not obey the simultaneity requirement. I work out two examples: the Einstein-de Sitter model, in which space, for an inertial observer, is not flat nor homogeneous, and the de Sitter case.
\end{abstract}

Key words. cosmology: muscellaneous - cosmology: theory

\section{Introduction}

General relativity and relativistic cosmology consider space-time as the arena for physics and it is an old question to define space and time. These notions are not covariant and all problems of general relativity and cosmology can be addressed without them, so that they may appear as rather academic (this is equivalent to the choice of a global reference frame). However, on the one hand, the literature refers often to space, for instance when it is affirmed that space is flat (or not), or homogeneous (or not) in a given cosmological model. On the other hand, quantum physics, or its interpretation, requires most often a splitting of space-time into space and time. This points out the necessity of a convenient definition of space in space-time, or equivalently, the choice of a convenient reference frame. The simple example of two inertial observers in Minkowski spacetime, with different velocities, shows that such a definition must be observer-dependent.

An observer needs a frame to do physics in his environment. Although space is defined locally without ambiguity (by orthogonality to his worldline, i.e., to his velocity $\boldsymbol{u}$ ), there are many different ways to extend this definition, i.e., to choose a frame beyond a neighborhood. Since

\footnotetext{
* e-mail: marclr@discovery.saclay.cea.fr
}

many experiments (e.g., synchronisation procedures) involve the observer at different moments of his history, a global frame is constrained to have convenient properties along his world line $O$, or at least a part of it (like being Minkowskian there). But, again, this is far from being sufficient to determine the choice. This paper provides a prescription which associates to any observer, defined by his world line, a unique reference frame with special properties.

A reference frame involves, as a first step, a family of spatial hypersurfaces orthogonal to $O$, which can be chosen in many different ways. Among popular prescriptions, the Fermi coordinates require the hypersurfaces to be generated by the spacelike geodesics orthogonal to $O$. But, as it is well known, these spatial surfaces may intersect, even in simple situations like for the Langevin observer (see below). This forbids a definition of time valid far from $O$ (different values of time would be associated with the same event). In cosmology, another popular choice is to select the orthogonal spatial hypersurfaces of homogeneity. But such hypersurfaces do not exist in all space-times, and the choice is clearly not convenient when the observer himself breaks the spatial symmetries (by his acceleration or rotation for instance). The prescription proposed here does not suffer from these drawbacks. In addition, it is the only one to obey the "simultaneity criterion", absent 
in all other prescriptions (except in the immediate neighborhood of $O$ ): the different points of "space" are seen as simultaneous (see below for a pecise definition) by the observer. Moreover, it has the additional advantage depending only on the conformal structure of the metric (except for the proper time of the observer), and its validity is broader than those mentioned above (for instance, in the absence of spatial homogeneity).

This prescription has in fact already been applied de facto in some circumstances, like the Rindler coordinates. But its validity is much more general, without having too restrictive conditions: for any observer (inertial or not), in any space-time (with some conditions, see below), it defines uniquely a "simultaneity space" (shortly, space) $\Sigma_{\tau}$ at any moment $\tau$. The $\Sigma_{\tau}$ do not intersect (even in the situations where the Fermi hypersurfaces do, like for instance for the Langevin observer, see below), and they are defined even in the absence of spatial homogeneity. This allows us to extend the validity of the observer's proper time to the whole space-time. In the FriedmannLemaître models, space so defined does not coincide with the intuitive idea of what space could be.

Numerous attempts to define a quantization procedure in curved space-time, and/or for non-inertial observers (see, e.g., Birrel \& Davis 1982), involve, more or less explicitly, a space-time splitting of space-time. This is especially important for giving a physical interpretation of quantum states in terms of frequencies or particles. For instance, I show below that the current interpretation of the Unruh effect, based on the Rindler coordinates, corresponds in fact to the prescription promoted here.

I emphasize that all quantities introduced in this work are covariant. This includes all the observer-dependent quantities like his velocity, acceleration, world-line and the special reference frame introduced here. An observable quantity (e.g., the energy) is a combination of a covariant quantity associated with the observer (e.g., its velocity $\boldsymbol{u})$ with a covariant quantity associated with the observed system (e.g., its momentum-energy tensor). The definition of a global frame associated with an observer may allow us to properly define non local quantities related to him.

Since the goal of this work is to construct a reference frame for an observer in a cosmological situation, one only requires its validity in the part of space-time which is causally related to him: all events considered here are causally linked to the observer. Throughout this paper, by an abuse of langage, I will call "space-time" the set $\mathcal{M}$, of events inside the particle horizon and the event horizon of the observer, if they exist. This first restriction applies to any construction of a global reference frame. Second, the synchronicity arguments require that null geodesics admit no conjugate points. This excludes the presence of gravitational lensing (also incompatible with the other prescriptions). Since this analysis concerns an observer in a cosmological situation (convenient for quantization), or for studying the perturbative development of irregularities, this condition is not too restrictive (I emphasize again that the validity of this approach is broader than for concurrent proposals).

In Sect. 2, I will implement the definition of simultaneity space, and the related notions. I show how they allow us to define a global frame convenient to the observer, and a congruence of associated observers. Section 3 applies these results to the inertial and Langevin observers in Minkowski spacetime. Section 4 considers the Rindler observer in Minkowski spacetime, and the associated Unruh effect. Section 5 considers the Friedmann-Lemaître cosmological models.

\section{A global reference frame for observers}

\subsection{The accelerated observer}

The most general observer is defined as a timelike world line $O(\tau)$ parametrized by proper time $\tau$. This defines its velocity $\boldsymbol{u}(\tau)$ and its acceleration $\boldsymbol{a}(\tau):=\nabla_{\tau} \boldsymbol{u}$ everywhere on his world-line. I call $\hat{\boldsymbol{a}}$ the unit vector parallel (and in the same direction) to the acceleration:

$\boldsymbol{a}:=\frac{\mathrm{d} \boldsymbol{u}}{\mathrm{d} \tau}=\hat{\boldsymbol{a}}|\boldsymbol{a}|$

Herereafter I consider only non rotating observers for which $\nabla_{\tau} \hat{\boldsymbol{a}}:=\nabla_{\tau} \hat{\boldsymbol{e}}_{1}=|\boldsymbol{a}| \boldsymbol{u}$ (the procedure here applies to the case with rotation, which will be considered in a subsequent paper). Transport along the world line corresponds to a Lorentz rotation (in the plane $\boldsymbol{u}, \boldsymbol{a}$ ).

Defining $\hat{\boldsymbol{e}}_{0}(\tau)=\boldsymbol{u}(\tau)$ and $\hat{\boldsymbol{e}}_{1}(\tau)=\hat{\boldsymbol{a}}(\tau)$, the transport is expressed by

$\nabla_{\tau} \hat{\boldsymbol{e}}_{A}=\Omega \cdot \hat{\boldsymbol{e}}_{A} \quad(A=1,2)$,

where $(\Omega \cdot V)^{\mu}:=\boldsymbol{a}^{[\mu} \boldsymbol{u}^{\nu]} V_{\nu}$ for the observer without (spatial) rotation.

We can naturally associate two different orthonormal frames to the non-rotating observer, along his world line. First, there is a parallely-transported frame $E$ but, for a non-inertial observer, no vector of this frame coincides with the velocity. Second, there is a Fermi-transported frame $f$ such that $f_{0}:=\boldsymbol{u}$. Since there is no spatial rotation, $f_{1}=\boldsymbol{a} /|\boldsymbol{a}|$ and the law $\nabla_{\tau} \hat{\boldsymbol{e}}_{A}=\Omega \cdot \hat{\boldsymbol{e}}_{A}$ is verified for $A=0,1,2,3$.

For non-rotating observers, the spatial sections (or spaces) keep axial symmetry around the unique spatial direction defined by the acceleration vector. For inertial observers (mainly considered in this paper, except in Sects. 3.1 and 4), the problem is purely 2-dimensional, with spatial spherical symmetry. The general (4-dimensional) case, including the rotating observer, will be examined in a subsequent paper (most of the calculations presented here will remain valid).

\subsection{Definition of space}

A given space-time admits, in general, many time-like foliations with associated space-like sections, compatible with the world-line of a given observer $O$. I will show that the 
requirement of synchronicity allows us to select a unique one, and thus provides a special global definition of space. This will require us to consider the whole story of the observer, including his future. In the following, I will assume that the causal structure of space-time admits only light cones without foldings and conjugate points. These restrictions are however appropriate for cosmology, and characterize a background space-time convenient for quantization. This excludes also multi-connected space-times. In the whole Paper I denote $\tilde{\boldsymbol{v}}$ the one-form metric-dual to a vector $\boldsymbol{v}$, i.e., such that $\langle\tilde{\boldsymbol{v}}, \boldsymbol{v}\rangle=g(\boldsymbol{v}, \boldsymbol{v}):=|\boldsymbol{v}|^{2}$.

It is well known that it is impossible to define absolute simultaneity in special or general relativity. However, a local prescription of simultaneity or, better, synchronicity, from the point of view of an observer is widely used (see, e.g., Landau \& Lifshitz 1966), and defines a local space-time splitting for this observer. The construction of space in this work is based on the extension of this prescription beyond a local neighborhood, with arguments of synchronicity which have a perfectly operational character.

Given an observer $O$, I define $\Sigma_{\tau}$, the hypersurface of synchronicity (HS) of $O$ at proper time $\tau$, as the set of events related by a null geodesics to both $O(\tau+\delta)$ and $O(\tau-\delta)$, where $\delta$ is an arbitrary interval of proper time for $O$ :

$\Sigma_{\tau}=\cup_{\delta}\left[I^{\text {future }}(\tau-\delta) \cap I^{\text {past }}(\tau+\delta)\right]$,

where $I^{\text {past }}(\tau)$ [resp. $I^{\text {future }}(\tau)$ ] denotes the null past [future] light-cone of the observer at proper time $\tau$.

Given the restrictions above, the surfaces $\Sigma_{\tau}$ for different values of $\tau$ completely fill $\mathcal{M}_{1}$. This allows to extend the vector field $\boldsymbol{u}$ to the totality of $\mathcal{M}$, by requiring that it is everywhere unit $(\boldsymbol{u} \cdot \boldsymbol{u}=1)$ and orthogonal to $\Sigma(\tau)$ (Fig. 1). The vector field $\boldsymbol{u}$ constitutes a foliation of $\mathcal{M}$, with the $\Sigma_{\tau}$ as transverse (orthogonal) surfaces. Each integral line of $\boldsymbol{u}$ will be labelled by its intersection $\boldsymbol{R}$ with $\Sigma(1)$ (for instance), so that any point of $\mathcal{M}$, can be written $(\tau, \boldsymbol{R})$. Practically, $R:=|\boldsymbol{R}|$ (evaluated in the spacelike surface $\Sigma_{\tau}$, see below) can be seen as half the interval of proper time between the emission of a flash light which illuminates a cosmic object at $x$, and the observation of the resulting image; or, equivalently, as half the interval of proper time between the emission of a light ray (or radar signal) by an observer, and its reception after mirror reflection by the object at $x$. The requirement of synchronicity imposes that "the space for $O(\tau)$ " is $\Sigma_{\tau}$.

Smarr \& York (1978), among others, have considered space-time slicing of space-time associated with a congruence of observers, that they call a kinematics of spacetime. The foliation introduced here is of this kind, associated with the congruence of "associated observers". However, since the latter are defined from the world line of the unique observer $O$, this procedure defines a unique space-time slicing, from the world line of this unique observer only. In a forthcoming paper, the properties of this "kinematics" will be discussed in more detail, and compared with other possibilities.

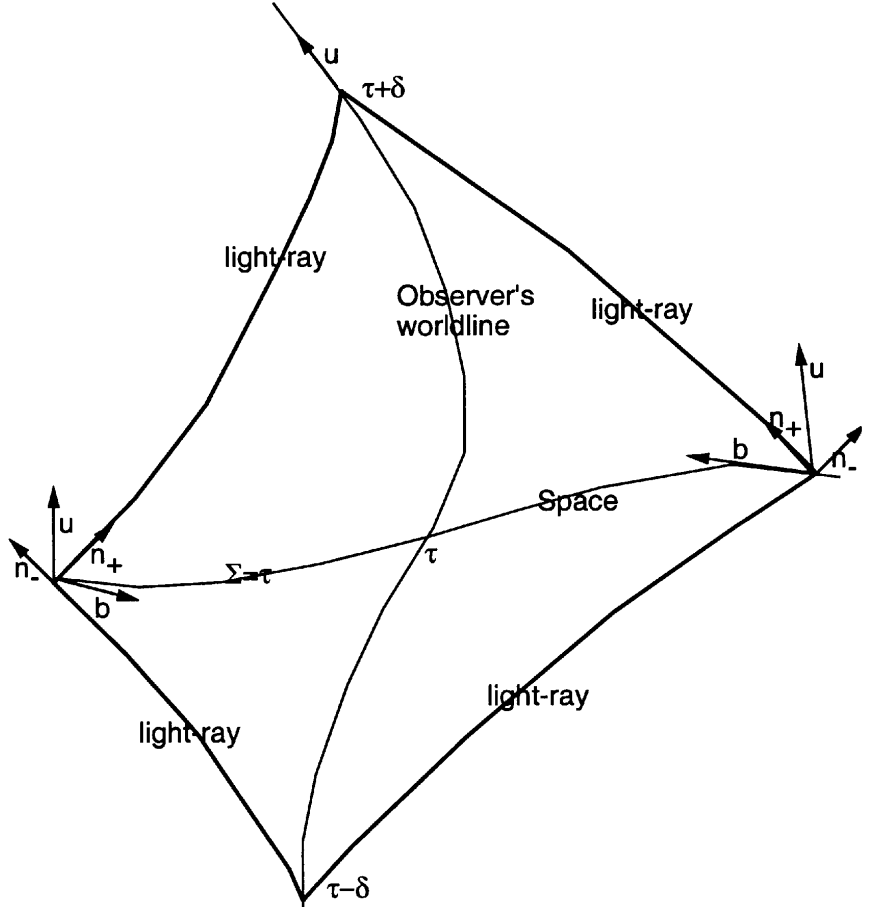

Fig. 1. At each point $x$, the light-rays from the observer (in the past and in the future) define the vectors $\boldsymbol{n}^{+}$and $\boldsymbol{n}^{-}$. The velocity $\boldsymbol{u}$ of the observer is (non parallely) transported to give the vectors $\boldsymbol{u}$ and $\boldsymbol{b}$ at $\boldsymbol{x}$.

The integral lines of $\boldsymbol{u}$ define a unique family of observers associated with $O$, that I will call the canonical observers associated with $O$. Care must be taken that they do not necessarily share the properties of $O$. For instance, they are not necessarily geodesic, even when $O$ is, when there is expansion. Along the word lines generated by $\boldsymbol{u}$ we will call the proper time $t$, with $t=\tau$ along $O$.

\subsection{Transport along the light-rays}

The properties of space are defined from those of the world-line of the observer, transported by the past and future light-rays. To explore them, we define the two null functions $\mathcal{N}_{-}(x)$ and $\mathcal{N}_{+}(x)$ such that the value of $\mathcal{N}_{-}(x)$ [resp. $\left.\mathcal{N}_{+}(x)\right]$ is the proper time $\tau-\delta$ of the observer $O$, when it emits a light-ray reaching $x$ [resp. $\tau+\delta$, when it receives a light-ray emitted from $x$ ]. In other words the null hypersurface $\mathcal{N}_{-}(x)=\tau$ [resp. $\mathcal{N}_{+}(x)=\tau$ ] is the future [resp. past] light cone of the observer at proper time $\tau$. We define their (null) (past and future) generators as $\tilde{\boldsymbol{n}}_{ \pm}=\nabla \mathcal{N}_{ \pm}=\mathrm{d} \mathcal{N}_{ \pm}$. Both are future directed, and normalized so that the frequency emitted or received by the observer is unity (see below).

It is easy to show that $\Sigma_{\tau}$ is defined by the equation

$T(x):=\left[\mathcal{N}_{-}(x)+\mathcal{N}_{+}(x)\right] / 2=\tau$.

For any point, $T$ constitutes a natural time-coordinate. In addition we define the deformed cylindric hypersurface

$R(x):=\left[\mathcal{N}_{+}(x)-\mathcal{N}_{-}(x)\right] / 2=\delta$ 
as the set of events at a constant "proper time interval" $\delta$ from the observer, when he describes its world-line. We define the proper time interval ( $P T$ interval) $\delta$ of an event $x$ as half the observer's proper time interval between the instants $\tau_{1}=\tau+\delta$ and $\tau_{2}=\tau-\delta$, when he receives and emits the light rays emitted and received by the event $x$. For any point, $R=|\boldsymbol{R}|$ defines a natural radial space coordinate.

Given the normalization above, we have

$\mathrm{d} T=\left(\tilde{\boldsymbol{n}}_{-}+\tilde{\boldsymbol{n}}_{+}\right) / 2$ and $\mathrm{d} R=\left(\tilde{\boldsymbol{n}}_{+}-\tilde{\boldsymbol{n}}_{-}\right) / 2$.

It is easy to check that $\mathrm{d} T \cdot \mathrm{d} R=0$, and

$\mathrm{d} T \cdot \mathrm{d} T=-\mathrm{d} R \cdot \mathrm{d} R:=N^{-2}=\boldsymbol{n}_{+} \cdot \boldsymbol{n}_{-} / 2$,

which defines the lapse function $N$ associated with this foliation. Since $\mathrm{d} T$ is orthogonal to $\Sigma$, we have $\tilde{\boldsymbol{u}}=N \mathrm{~d} T$. From $\boldsymbol{u}^{2}=1$, we have $N \boldsymbol{u} \cdot \mathrm{d} T=1$. Since, along $\mathcal{O}$, $\tau=T$, this implies $N=1$ on $\mathcal{O}$.

Everywhere (except on $\mathcal{O}$ ), we define

$\tilde{\boldsymbol{b}}:=N \mathrm{~d} R=\tilde{\boldsymbol{u}}-N \tilde{\boldsymbol{n}}_{-}=-\tilde{\boldsymbol{u}}+N \tilde{\boldsymbol{n}}_{+}$.

We have $\boldsymbol{b}^{2}=-1, \boldsymbol{u} \cdot \boldsymbol{b}=0, \boldsymbol{u}+\boldsymbol{b}=N \boldsymbol{n}_{+}$and $\boldsymbol{u}-\boldsymbol{b}=$ $N \boldsymbol{n}_{-}$. Thus, $\boldsymbol{b}$ is a unit-space-like vector, tangent to $\Sigma_{\tau}$ and, in some sense, pointing towards the observer $O$. In general, the vector $\boldsymbol{b}$ is not geodesic but it is chorodesic, due to the synchronicity property and the congruence of associated observers is quasi-rigid (see Bel 1998).

\subsection{Associated observers}

The vector field $\boldsymbol{u}$ is perfectly defined everywhere and characterizes the family of canonical observers. This family defines a "kinematics" in the sense of Smarr \& York (1978). All the relevant formalism of projectors, lapse and shift functions (the latter being zero here, see below), intrinsic curvature, etc., applies and will be discussed in a forthcoming paper.

The vector fields $\boldsymbol{u}$ and $\boldsymbol{b}$ are not transported parallely along the light rays $(\boldsymbol{u}$ is not parallely transported along itself, in general). To have a clearer view, it is convenient to introduce the two vector fields $\boldsymbol{U}^{+}$and $\boldsymbol{U}^{-}$which are, by definition, parallely transported along $\boldsymbol{n}^{+}$and $\boldsymbol{n}^{-}$respectively, $\boldsymbol{n}^{\varepsilon} \cdot \nabla \boldsymbol{U}^{\varepsilon}=0$, and which both coincide with $\boldsymbol{u}$ along the world line of $O$ (Fig. 2). These requirements are sufficient to define them everywhere. Both are (time-like) unitary and, when $O$ is inertial, the spherical symmetry allows to developed them as

$\boldsymbol{U}^{\varepsilon}=\cosh \phi \boldsymbol{u}+\varepsilon \sinh \phi \boldsymbol{b}$.

In the same way that we introduced the unit vector field $\boldsymbol{b}$ orthogonal to $\boldsymbol{u}$ and in the plane defined by the light-rays, we can introduce two unit vector fields $\boldsymbol{B}^{+}$and $\boldsymbol{B}^{-}$, orthogonal to $\boldsymbol{U}^{+}$and $\boldsymbol{U}^{-}$, and in the same plane. What is interesting here is that the values of $\boldsymbol{U}^{+}$and $\boldsymbol{B}^{+}$, at any point $x$, depend only (via the transport along the light ray $\boldsymbol{n}^{+}$) on the velocity of the observer $O$ at the value $T(x)+R(x)$ of its proper time. For this reason, we could

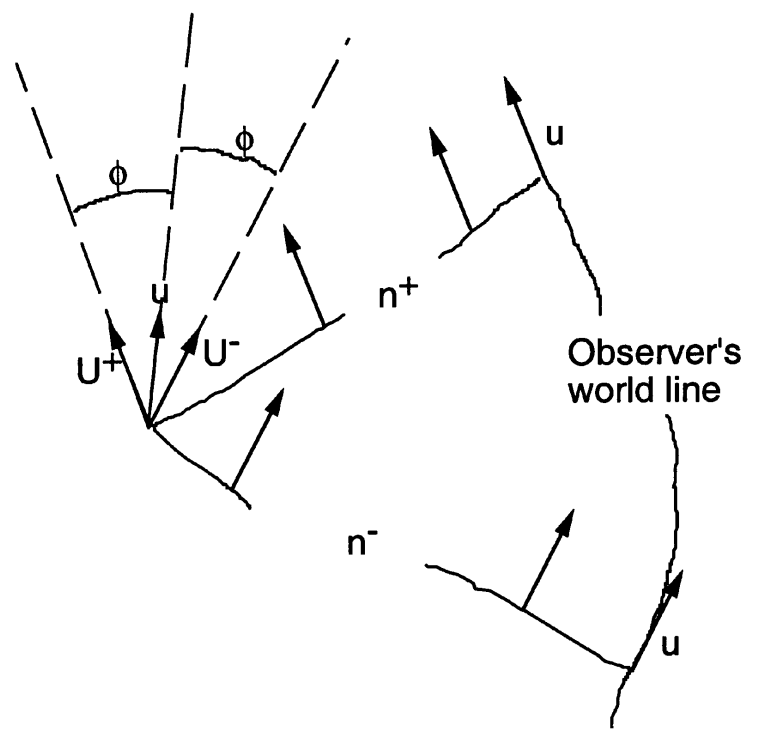

Fig. 2. The field $\boldsymbol{u}$ is not parallely transported. At each point $x, \boldsymbol{U}^{+}$is defined by the parallel transport of $\boldsymbol{u}$ from the future, i.e., by $\boldsymbol{n}^{+}, \boldsymbol{U}^{-}$is defined by the parallel transport of $\boldsymbol{u}$ from the past, i.e., by $\boldsymbol{n}^{-}$.

call $\left(\boldsymbol{U}^{+}, \boldsymbol{B}^{+}\right)$the "future frame". Similarly, $\boldsymbol{U}^{-}(x)$ and $\boldsymbol{B}^{-}(x)$, the "past frame", depend only on the velocity of $O$ at the value $T(x)-R(x)$ of its proper time. The two frames at point $x$ are transformed into each other by a Lorentz rotation of (hyperbolic) angle $2 \phi$ and the frame $[\boldsymbol{u}(x), \boldsymbol{b}(x)]$ is a "bisector" frame, obtained from the previous ones by hyperbolic rotations of angles $\phi$ and $-\phi$ in the $\left(\boldsymbol{n}^{+}, \boldsymbol{n}^{-}\right)$plane: the value $\boldsymbol{u}(x)$ can be reconstructed as

$\boldsymbol{u}=\frac{\boldsymbol{U}^{+}+\boldsymbol{U}^{-}}{\sqrt{2\left(1+\boldsymbol{U}^{+} \cdot \boldsymbol{U}^{-}\right)}}$.

The geodesic equation $\boldsymbol{n}^{+} \cdot \nabla \boldsymbol{n}^{+}=0$ leads to $\boldsymbol{n}^{+}$. $\nabla\left(\ln N^{-1}\right)=\boldsymbol{n}^{+} \cdot \nabla \phi$ and, after integration along the light-ray,

$\phi(x)=-\ln N(x)$.

Finally, $\boldsymbol{U}^{\varepsilon}=\left[\boldsymbol{n}^{\varepsilon}+N^{2} \boldsymbol{n}^{(-\varepsilon)}\right] / 2$. Note that $\boldsymbol{U}^{\varepsilon}$ may be used like $\boldsymbol{u}$ to construct spacelike hypersurfaces and reference frames. For instance, Marzlin (1994) proposed a reference frame based on $\boldsymbol{U}^{+}$, but which does not obey synchronicity.

This example of a bisector frame, in relation to two other frames, may be extended to provide a local surface of synchronicity for two different observers (see Fig. 5 of Ali et al. 1990), useful for the study of the quantum evolution of two interacting particles in space-time (Ali et al. 1990). This will be further developed in a subsequent paper.

There is a closed path starting from the arbitrary point $x$, going along the light-ray $\boldsymbol{n}^{+}$to the (inertial) observer, then going back to the past of the observer along his worldline, and back to the point $x$ along the light-ray $\boldsymbol{n}^{-}$. When the observer is inertial, $\boldsymbol{u}$ is parallely transported along his world-line, so that the transport of $\boldsymbol{U}^{+}$, then $\boldsymbol{u}$ and then 
of $\boldsymbol{U}^{-}$remains parallel. This angular deficit of $2 \phi$, along a closed path in the sheet $\mathcal{D}$ (see below) is a mark of the average curvature of space-time there.

\section{Redshifts}

Let us consider a congruence of (non necessarily canonical) objects with velocity $\boldsymbol{V}(x)$ at the point (event) $x$ in space-time. An object at $x$ is seen with redshift $z^{+}=\left(\boldsymbol{n}^{+}(x) \cdot \boldsymbol{V}(x)\right)^{-1}$ by the observer (in the future) and sees the observer (in his past) with a redshift $z^{-}=$ $\boldsymbol{n}^{-}(x) \cdot \boldsymbol{V}(x)$. For the congruence of canonical observers, $z^{+}=N(x)$ and $z^{-}=N(x)^{-1}$. These observers are comoving with respect to the coordinate $R$, i.e., they keep a constant value of $R$. On the other hand, there is a unique congruence of objects for which $z^{+}=1$ [resp. $z^{-}=1$ ], those with velocity $\boldsymbol{U}^{+}$[resp. $\left.\boldsymbol{U}^{-}\right]$.

\section{Frames}

Since we assume that the observer is non-rotating, the problem has a spatial axial symmetry around the direction of his acceleration (and even spherical symmetry if the observer is inertial, i.e., without acceleration).

At each point $x \in \mathcal{M}$ (except on $O$ ), the two vectors $\boldsymbol{n}_{+}(x)$ and $\boldsymbol{n}_{-}(x)$, or $\boldsymbol{u}(x)$ and $\boldsymbol{b}(x)$, or $\boldsymbol{U}^{+}(x)$ and $\boldsymbol{U}^{-}(x)$ define the same plane $D_{x}$ in the tangent space $T_{x}$. These planes generate a foliation with integral surfaces $\mathcal{D}$ (Fig. 3). Each surface $\mathcal{D}$ is generated, with time, by a constant spatial direction $(\alpha, \beta)$ from the point of view of the observer. In each $\mathcal{D}$ (itself parametrized by $(\alpha, \beta)$ ), we will use $R$ as a spatial (radial) coordinate and $T$ as a temporal coordinate. Thus any point $x=(T, R, \alpha, \beta)$. By definition, a light ray from [to] a point $(T, R, \alpha, \beta)$ reaches [resp. starts from] the observer at proper time $T+R$ [resp. $T-R]$. The coframe $(\mathrm{d} T, \mathrm{~d} R)$ has for a dual frame $\left(\boldsymbol{e}_{T}:=\partial_{T}, \boldsymbol{e}_{R}:=\partial_{R}\right)$. These two vectors generate the surface $\mathcal{D}$. From

$<\mathrm{d} T, \boldsymbol{e}_{T}>=<\mathrm{d} R, \boldsymbol{e}_{R}>=1$,

$<\mathrm{d} R, \boldsymbol{e}_{T}>=<\mathrm{d} T, \boldsymbol{e}_{R}>=0$,

we can show $\boldsymbol{e}_{T}=N^{2}\left(\boldsymbol{n}_{+}+\boldsymbol{n}_{-}\right) / 2=N \boldsymbol{u}$ and $\boldsymbol{e}_{R}=$ $N^{2}\left(\boldsymbol{n}_{+}-\boldsymbol{n}_{-}\right) / 2=-N \boldsymbol{b}$. The two vectors commute and thus they do Lie-transport each other: $\boldsymbol{e}_{T} \cdot \nabla \boldsymbol{e}_{R}=$ $\boldsymbol{e}_{R} \cdot \nabla \boldsymbol{e}_{T}$. This is the same for the two vectors $N^{2} \boldsymbol{n}^{+}$ and $N^{2} \boldsymbol{n}^{-}$.

The space-like plane $C_{x}$ orthogonal to all these vectors is tangent to the past and future light cones at this point, and also to the hypersurface $\Sigma$ at this point. It is the tangent plane to the surface

$\mathcal{C}=\mathrm{I}^{+} \cap \mathrm{I}^{-}=\mathrm{I}^{+} \cap \Sigma$.

This deformed 2-sphere $\mathcal{C}(\tau, \delta)$ is the set of all the points at equal $P T$-interval $\delta$ from the observer at the moment $\tau$, i.e., the set of all points of the hypersurface $\Sigma_{\tau}$ at equal $P T$-interval. Both $\boldsymbol{u}$ and $\boldsymbol{b}$ are orthogonal to $\mathcal{C}$.

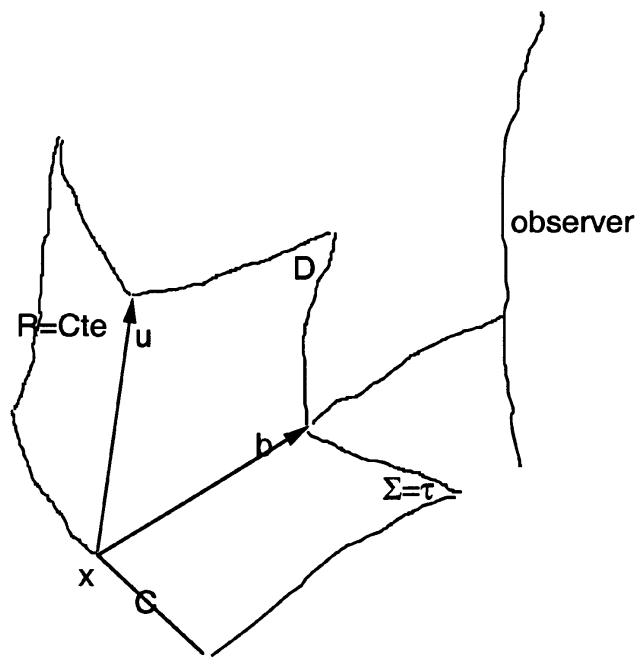

Fig. 3. At each point $x, \boldsymbol{u}$ and $\boldsymbol{b}$ are tangent to the surface $\mathcal{D}$. The curve $C$ (tangent to $\boldsymbol{b}$ is contained in space $\Sigma_{\tau}$ and in the "cylinder" $R=C^{\text {te }}$.

We will choose two orthogonal unit space-like vectors $\boldsymbol{e}_{3}$ and $\boldsymbol{e}_{4}$ in $C$ (many choices are possible; since we only consider non rotating observers, the problem is essentially two-dimensional and we do not care about the choice of $\boldsymbol{e}_{3}$ and $\boldsymbol{e}_{4}$; this will be the subject of a forthcoming paper). $\boldsymbol{e}_{3}$ and $\boldsymbol{e}_{4}$ form a basis for $C$, tangent to the 2 -surface $\mathcal{C}$. Any of the previous pair with these two vectors form a (pseudo-ON or ON) basis of the tangent space to spacetime. Also, $\boldsymbol{n}^{+}$or $\boldsymbol{n}^{-}, \boldsymbol{e}_{3}$ and $\boldsymbol{e}_{4}$ form a pseudo-ON basis for the tangent space to the light cones.

\section{The metric}

Since $\boldsymbol{u}$ and $\boldsymbol{b}$ are orthogonal and unitary, the metric can be written

$$
\begin{aligned}
\mathrm{d} s^{2} & =\tilde{\boldsymbol{u}}^{2}-\tilde{\boldsymbol{b}}^{2}-\left(\boldsymbol{e}^{3}\right)^{2}-\left(\boldsymbol{e}^{4}\right)^{2} \\
& =N^{2}\left(\mathrm{~d} T^{2}-\mathrm{d} R^{2}\right)-\left(\boldsymbol{e}^{3}\right)^{2}-\left(\boldsymbol{e}^{4}\right)^{2}
\end{aligned}
$$

where $-\left(\boldsymbol{e}^{3}\right)^{2}=g_{\alpha \alpha} \mathrm{d} \alpha^{2}$ and $-\left(\boldsymbol{e}^{4}\right)^{2}=g_{\beta \beta} \mathrm{d} \beta^{2}$. Thus, $N$ appears as the lapse function associated with the foliation, or to the congruence of associated observers (the shift vector being zero). Since $N=1$ at the position of $O$, the metric is locally Minkowskian for him, everywhere on his worldline. The usual ADM formalism allows to define time and space projectors, as well as the fundamental forms (metric and extrinsic curvature) on the surfaces $\Sigma_{\tau}$ (see, e.g., Smarr \& York 1978).

As we have said, $T, R$ (with appropriate angular coordinates) constitute a well-behaved system of coordinates and provide a global reference frame in $\mathcal{M}$. Written in these coordinates the coefficients of the metric reduce to $\pm N^{2}$. But $N=1$ on $O$ and is stationary (by spherical symmetry arguments) at $O$ if the observer is inertial. Thus, when the observer is inertial, $T$ and $R$ are normal coordinates based on any point of his world line. 


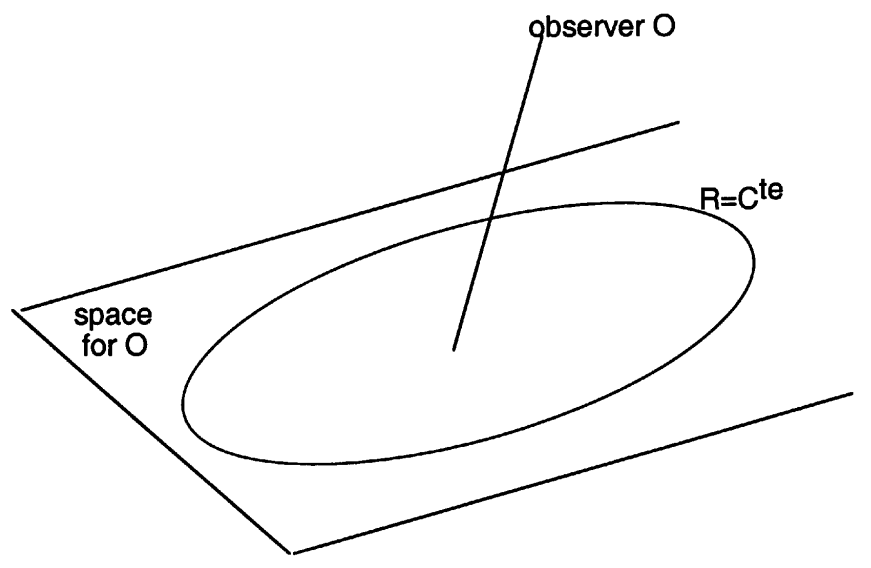

Fig. 4. For the inertial observer in Minkowski spacetime, with arbitrary velocity (rapidity $\psi$ ), space is the hyperplane with inclination $\psi$. We have drawn a curve $R=C^{\text {te }}$, in this plane.

We point out that $T$ and $R$ are not, in general, Gaussian normal coordinates since $T$ is not a proper time, except on $O$ (Gaussian normal coordinates cannot, in general, be constructed for this foliation). But they have the advantage that the surfaces of constant "time" $T$ are global surfaces of simultaneity, although the surfaces of constant Gaussian normal coordinates are not (they verify only local simultaneity).

Now I will consider some special cases.

\section{The inertial observer in Minkowski spacetime}

The inertial observer $O$ has zero acceleration and there is no cosmic expansion. Its velocity is defined by

$u^{0}=c, u^{1}=s, u^{2}=u^{3}=0$,

where $c:=\cosh \psi$ and $s:=\sinh \psi$, the rapidity $\psi$ being a constant. Its world line is $x^{0}=c \tau, x^{1}=s \tau, x^{2}=x^{3}=0$. A light-ray passing through $x$ reaches $O$ at proper time $\tau$ such that

$\left(x^{0}-c \tau\right)^{2}=\left(x^{1}-s \tau\right)^{2}+\left(x^{2}\right)^{2}+\left(x^{3}\right)^{2}$.

For the arbitrary point $x$, it is convenient to define $x^{0}=$ $\Delta \cosh \beta, x^{1}=\Delta \sinh \beta$, so that the solutions of this equation give

$\begin{aligned} \mathcal{N}_{\varepsilon}= & \Delta \cosh (\psi-\beta) \\ & +\varepsilon \sqrt{\Delta^{2} \sinh (\psi-\beta)^{2}+\left(x^{2}\right)^{2}+\left(x^{3}\right)^{2}}\end{aligned}$

$T=\Delta \cosh (\psi-\beta)=c x^{0}-s x^{1}$,

$$
\begin{aligned}
R & =\sqrt{\Delta^{2} \sinh (\psi-\beta)^{2}+\left(x^{2}\right)^{2}+\left(x^{3}\right)^{2}} \\
& =\sqrt{\left(s x^{0}-c x^{1}\right)^{2}+\left(x^{2}\right)^{2}+\left(x^{3}\right)^{2}}
\end{aligned}
$$

and $N=1$.

The surface $\Sigma_{\tau}$ is the plane of equation $c x^{0}-s x^{1}=$ $\tau$, inclined by $\psi$ with respect to the vertical, and thus

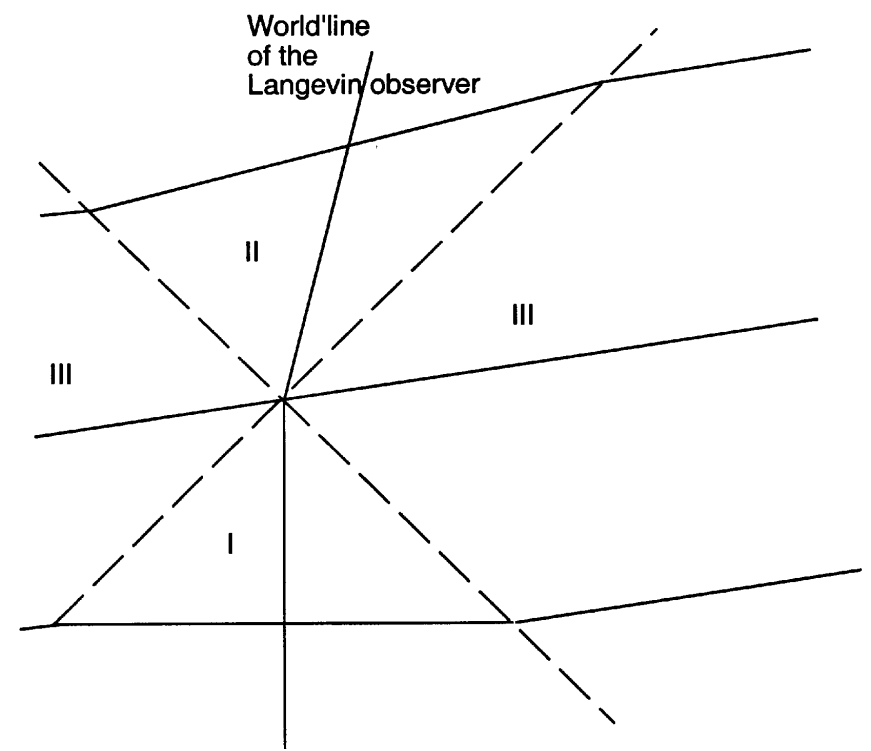

Fig. 5. World's line and (cuts of) space at various moments for the Langevin observer. Its line cone is indicated by the dashed lines.

orthogonal to the world line of the inertial observer. Thus, space is different for all inertial observers.

Finally, $u=c \mathrm{~d} x^{0}-s \mathrm{~d} x^{1}$ and

$$
\begin{aligned}
\tilde{\boldsymbol{b}}= & \frac{1}{R}\left[\left(s x^{0}-c x^{1}\right)\left(s \mathrm{~d} x^{0}-c \mathrm{~d} x^{1}\right)\right. \\
& \left.+x^{2} \mathrm{~d} x^{2}+x^{3} \mathrm{~d} x^{3}\right]
\end{aligned}
$$

points towards the observer at time $\tau=T(x)$. In the space $\Sigma_{\tau}$ (Fig. 4), it is natural to define the coordinate $y:=$ $-s x^{0}+c x^{1}=y / c$ so that $R^{2}=y^{2}+\left(x^{2}\right)^{2}+\left(x^{3}\right)^{2}$ and the spatial metric $-\mathrm{d} s^{2}=\mathrm{d} y^{2}+\left(\mathrm{d} x^{2}\right)^{2}+\left(\mathrm{d} x^{3}\right)^{2}$.

\subsection{The Langevin observer in Minkowski spacetime}

It is well known that the solution of the celebrated "Langevin's twin paradox" lies in geometry. I define a Langevin observer as an inertial observer which is initially inertial, then suffers an instantaneous acceleration, and then is inertial again (Fig. 5). Such an observer is able to meet his twin, who remained always inertial, with a different lapse of proper time. Is is often quoted (see, e.g., Misner et al. 1973) that it is impossible to define space globally for such an observer. Here I show that the synchronicity prescription applies perfectly and provides an unambiguous definition of space for this observer. Thus I define the space-time trajectory of this observer as

$x^{0}=\tau, x^{1}=x^{2}=x^{3}=0, \quad$ for $\quad t<0$,

$x^{0}=c \tau, x^{1}=s \tau, x^{2}=x^{3}=0, \quad$ for $\quad t>0$,

with $c:=\cosh \psi$ and $s:=\sinh \psi$.

The light cone of the observer at $t=0, \mathcal{L}^{0}$, divides the space-time into three parts I, II and III (see Fig. 5) corresponding to the past, future and spatially related regions 
to the acceleration point. Studying the light-rays from [to] the point $x$ to [from] the observer gives the functions:

\section{- Region I}

$$
\begin{aligned}
& \mathcal{N}^{\varepsilon}(x)=x^{0}+\varepsilon \sqrt{\left(x^{1}\right)^{2}+\left(x^{2}\right)^{2}+\left(x^{3}\right)^{2}}, \\
& T(x)=t, R(x)=\sqrt{\left(x^{1}\right)^{2}+\left(x^{2}\right)^{2}+\left(x^{3}\right)^{2}} .
\end{aligned}
$$

\section{- Region II}

$$
\begin{aligned}
\mathcal{N}^{\varepsilon}(x) & =c x^{0}-s x^{1}+\frac{\varepsilon}{c} \\
\times & \sqrt{\left(c x^{0}-s x^{1}\right)^{2}-\left(x^{0}\right)^{2}+\left(x^{1}\right)^{2}+\left(x^{2}\right)^{2}+\left(x^{3}\right)^{2}} \\
& =c x^{0}-s x^{1}+\frac{\varepsilon}{c} \\
\times & \sqrt{\left(s x^{0}-c x^{1}\right)^{2}+\left(x^{2}\right)^{2}+\left(x^{3}\right)^{2}} \\
T(x) & =c x^{0}-s x^{1}, \\
R(x) & =\sqrt{\left(c x^{0}-s x^{1}\right)^{2}-\left(x^{0}\right)^{2}+\left(x^{1}\right)^{2}+\left(x^{2}\right)^{2}+\left(x^{3}\right)^{2}} \\
& =\sqrt{\left(s x^{0}-c x^{1}\right)^{2}+\left(x^{2}\right)^{2}+\left(x^{3}\right)^{2}} .
\end{aligned}
$$

\section{- Region III}

$$
\begin{aligned}
\mathcal{N}^{+}(x)= & c x^{0}-s x^{1} \\
& +\sqrt{\left(s x^{0}-c x^{1}\right)^{2}+\left(x^{2}\right)^{2}+\left(x^{3}\right)^{2}}, \\
\mathcal{N}^{-}(x)= & x^{0}-\sqrt{\left(x^{1}\right)^{2}+\left(x^{2}\right)^{2}+\left(x^{3}\right)^{2}} \\
2 T(x)= & x^{0}+c x^{0}-s x^{1} \\
& +\sqrt{\left(s x^{0}-c x^{1}\right)^{2}+\left(x^{2}\right)^{2}+\left(x^{3}\right)^{2}} \\
& -\sqrt{\left(x^{1}\right)^{2}+\left(x^{2}\right)^{2}+\left(x^{3}\right)^{2}}
\end{aligned}
$$

and

$$
\begin{aligned}
2 R(x)= & -x^{0}+c x^{0}-s x^{1} \\
& +\sqrt{\left(s x^{0}-c x^{1}\right)^{2}+\left(x^{2}\right)^{2}+\left(x^{3}\right)^{2}} \\
& +\sqrt{\left(x^{1}\right)^{2}+\left(x^{2}\right)^{2}+\left(x^{3}\right)^{2}} .
\end{aligned}
$$

The surface $\Sigma_{\tau}$ of equation $T(x)=\tau$ defines space for the observer at proper time $\tau$.

Let us consider its projection in the $\left(x^{0}, x^{1}\right)$ plane (Fig. 5):

\section{- Region I}

$x^{0}=\tau$.

This is a straight horizontal line, where $R(x)=x^{1}$.

\section{- Region II}

$c x^{0}-s x^{1}=\tau$

or

$\tau / \cosh \psi=x^{0}-\tanh \psi x^{1}$.

This is a line inclined of $\psi$ with respect to the vertical, and thus orthogonal to the world line of the observer in that region. In this line,

$$
\begin{aligned}
R(x) & =\sqrt{\left(c x^{0}-s x^{1}\right)^{2}-\left(x^{0}\right)^{2}+\left(x^{1}\right)^{2}} \\
& =c x^{1}-s x^{0} .
\end{aligned}
$$

\section{- Region III}

$$
\begin{aligned}
2 \tau= & x^{0}+c x^{0}-s x^{1} \\
& +\sqrt{\left(c x^{0}-s x^{1}\right)^{2}-\left(x^{0}\right)^{2}+\left(x^{1}\right)^{2}}-x^{1} \\
= & x^{0}+c x^{0}-s x^{1}+c x^{1}-s x^{0}-x^{1},
\end{aligned}
$$

or

$$
\frac{\tau \exp (\psi / 2)}{\cosh (\psi / 2)}=-x^{0}+x^{1} \tanh (\psi / 2) \text {. }
$$

This is a line inclined of $\psi / 2$ with respect to the vertical, i.e., at equal hyperbolic angle $\psi / 2$ of the two previous lines (Fig. 5). In this line,

$$
\begin{aligned}
2 R(x)= & -x^{0}+c x^{0}-s x^{1} \\
& +\sqrt{\left(c x^{0}-s x^{1}\right)^{2}-\left(x^{0}\right)^{2}+\left(x^{1}\right)^{2}}+x^{1} \\
= & -x^{0}+c x^{0}-s x^{1}+c x^{1}-s x^{0}+x^{1} .
\end{aligned}
$$

For the observer at an arbitrary moment, space is made of a plane circle $\mathrm{S}^{\mathrm{I}}$ [or $\mathrm{S}^{\mathrm{II}}$ ] up to the light cone $\mathcal{L}^{0}$ and is continued by a composite surface $S^{I I I}$ beyond. Except at the single moment when the observer experiences the instantaneous acceleration, space is not flat, nor homogeneous.

This is the simplest example where our prescription differs from the other ones. As it is well known, it is impossible to extend the Fermi coordinates outside the conical regions. And no homogeneous hypersurfaces would be convenient. Thus, in this simple case, our prescription is the only one providing a reference frame associated to the observer valid in the whole space-time, to extend the validity of his proper time, and to consider unambiguous synchronicity procedures (a similar conclusion has been reached by Dolby \& Gully 2001).

\section{The Rindler observer}

The Rindler observer in Minkowski spacetime has constant acceleration $a$. Its velocity is defined by

$u^{0}=\cosh (a \tau), \quad u^{1}=\sinh (a \tau), \quad u^{2}=u^{3}=0$,

with acceleration $a^{0}=a \sinh (a r), \quad a^{1}=$ a $\cosh (a \tau), a^{2}=a^{3}=0$ (with no loss of generality, I have taken the $x^{2}$ direction parallel to the acceleration). The world line has the equation $x^{0}=a^{-1} \sinh (a \tau), x^{1}=$ $a^{-1} \cosh (a \tau), x^{2}=x^{3}=0$, an hyperbola in space-time. A light-ray from (to) $x$ reach the observer at proper times $\tau$ such that

$$
\begin{aligned}
{\left[x^{0}-a^{-1} \sinh (a \tau)\right]^{2}=} & {\left[x^{1}-a^{-1} \cosh (a \tau)\right]^{2} } \\
& +\left(x^{2}\right)^{2}+\left(x^{3}\right)^{2} .
\end{aligned}
$$

Since (anticipating) the solution requires $\left(x^{1}\right)^{2}-\left(x^{0}\right)^{2}>0$, we can introduce the Rindler coordinates

$x^{0}:=a^{-1} \exp (a \xi) \sinh (a \eta)$ and

$x^{1}:=a^{-1} \exp (a \xi) \cosh (a \eta)$. 


\subsection{The problem in two dimensions}

I present first the problem in two dimensions, as it is usually treated, since it is particularly simple and pedagogic. I further treat the complete 4-dimensional problem.

The condition (33) becomes $\cosh \left(\begin{array}{ccc}a & -a & -a\end{array}\right)=$ $\cosh (a \xi)$, or $\tau-\eta= \pm \xi$. It results that

$\mathcal{N}_{\varepsilon}=\eta+\varepsilon|\xi|$,

$T=\eta, R=|\xi|$

and $N^{2}=a^{-2} \exp (2 a \xi)=\left[x^{2}-t^{2}\right]$, so that $N \mathrm{~d} N=$ $x \mathrm{~d} x-t \mathrm{~d} t=2 a^{-1} \exp (2 a \xi) \mathrm{d} \xi$.

The hypersurface (line) $\Sigma_{\tau}$ has the equation $\eta=\tau$, which implies $x^{0}=\tanh \tau x^{1}$, a straight line through the origin. The surfaces of constant PT-interval $\delta$ are the hyperbolae of equation $\xi=\delta$, or $\left(x^{1}\right)^{2}-\left(x^{0}\right)^{2}=$ $a^{-2} \exp (2 a \delta)$.

The forms

$\mathrm{d} T=\mathrm{d} \eta=\frac{x \mathrm{~d} t-t \mathrm{~d} x}{x^{2}-t^{2}}$

and

$\mathrm{d} R=\mathrm{d} \xi=\frac{-t \mathrm{~d} t+x \mathrm{~d} x}{x^{2}-t^{2}}$,

so that $\boldsymbol{n}^{\varepsilon}=\frac{\varepsilon \mathrm{d} t+\mathrm{d} x}{x+\varepsilon t}$.

\subsection{The four-dimensional problem}

The solutions of Eq. (33) are given by

$$
\begin{aligned}
\cosh (a \eta-a \tau)= & \cosh (a \xi)+a^{2} \exp (-a \xi)\left[\left(x^{2}\right)^{2}\right. \\
& \left.+\left(x^{3}\right)^{2}\right] / 2:=\cosh a \xi^{\prime},
\end{aligned}
$$

where

$\exp \left(a \xi^{\prime}\right):=\cosh (a \xi)+\exp (-a \xi) a^{2}\left[\left(x^{2}\right)^{2}+\left(x^{3}\right)^{2}\right] / 2$

$+\sqrt{\left(\cosh (a \xi)+\exp (-a \xi) a^{2}\left[\left(x^{2}\right)^{2}+\left(x^{3}\right)^{2}\right] / 2\right)^{2}-1}>1$.

This implies $\eta=\tau \pm \xi^{\prime}$, and thus,

$\mathcal{N}_{\varepsilon}=\eta+\varepsilon \xi^{\prime}$,

$T=\eta$,

$R=\xi^{\prime}$

The forms

$\mathrm{d} T=\mathrm{d} \eta=\frac{x^{1} \mathrm{~d} x^{0}-x^{0} \mathrm{~d} x^{1}}{\left(x^{1}\right)^{2}-\left(x^{0}\right)^{2}}$

and

$\mathrm{d} R=\mathrm{d} \xi^{\prime}=\mathrm{d} \xi=\frac{-x^{0} \mathrm{~d} x^{0}+x^{1} \mathrm{~d} x^{1}}{\left(x^{1}\right)^{2}-\left(x^{0}\right)^{2}}$,

so that $\boldsymbol{n}^{\varepsilon}=\frac{\varepsilon \mathrm{d} x^{0}+\mathrm{d} x^{1}}{x^{1}+\varepsilon x^{0}}$ and $N^{2}=a^{-2} \exp (2 a \xi)=$ $\left[\left(x^{1}\right)^{2}-\left(x^{0}\right)^{2}\right]$.
The hypersurface $\Sigma_{\tau}$ is the [flat] hyperplane through the origin of equation $x^{0}=\tanh \tau x^{1}$ (it has as projection the line seen in the previous section). The (spatial) metric on $\Sigma_{\tau}$ is given by

$$
\begin{aligned}
\mathrm{d} \sigma^{2} & =N^{2} \mathrm{~d} R^{2}+\left(\mathrm{d} x^{2}\right)^{2}+\left(\mathrm{d} x^{3}\right)^{2} \\
& =a^{-2}[\mathrm{~d} \exp (a \xi)]^{2}+\left(\mathrm{d} x^{2}\right)^{2}+\left(\mathrm{d} x^{3}\right)^{2} \\
& =\left(\mathrm{d} x^{1} / \cosh \eta\right)^{2}+\left(\mathrm{d} x^{2}\right)^{2}+\left(\mathrm{d} x^{3}\right)^{2},
\end{aligned}
$$

the latter form showing that its hypersurface is flat and homogeneous.

The surfaces of constant PT-interval $\delta$ are given by $\xi^{\prime}=\delta$, or

$2 a^{-1} \cosh (a \delta) \sqrt{\left(x^{1}\right)^{2}-\left(x^{0}\right)^{2}}-a^{-2}$

$$
=\left(x^{1}\right)^{2}-\left(x^{0}\right)^{2}+\left(x^{2}\right)^{2}+\left(x^{3}\right)^{2} .
$$

This calculation shows that the widely used Rindler coordinates correspond in fact to the definition of space and time introduced here for the accelerated observer, which justifies their use. This sheds some light on the Unruh effect, which appears as a consequence of the different space-time splittings for the two observers (inertial and Rindler): they associate different frequencies to the same state (e.g., the Minkowski inertial vacuum). This has led Pauri \& Vallisneri (1999) to invoque a classical (not quantum) origin for this effect, to be discussed further.

\section{The cosmological observer}

Turning to cosmology, I consider the FriedmannLemaître models, i.e., space-times with spatial sections of maximal symmetry. There exists a special system of coordinates in which the metric takes the form

$\mathrm{d} s^{2}=A(\eta)^{2}\left(\mathrm{~d} \eta-\left[\mathrm{d} \sigma^{2}-S(\sigma)^{2}\left(\mathrm{~d} \alpha^{2}+\sin ^{2} \alpha \mathrm{d} \beta^{2}\right)\right]\right)$

where $A$ is the usual scale factor, the expression between quotes is the metric of a spatial section with maximal symmetry (thus $\mathbb{R}^{3}, \mathrm{~S}^{3}$ or $\mathrm{H}^{3}$ ) and $\eta$ is the conformal time. Although different systems of coordinates would be as well convenient, I will perform calculations with the coordinates $(\eta, \sigma, \alpha, \beta)$.

I will consider only a cosmological inertial observer (CIO) $O_{I}$ which follows the line $\sigma=0$. The problem thus preserves spherical symmetry. All light-rays considered will be radial, and $\alpha, \beta$ will play no role. His proper time $\tau$ is defined by $\mathrm{d} \tau=A \mathrm{~d} \eta$. The functions $\eta(\tau)$ and its inverse $f$ such that $f[\eta(\tau)]:=\tau$ will play an important role. Since $\eta>0$, the CIO has a particle horizon and $\mathcal{M}$, is defined inside it, i.e., by $\sigma<\eta$.

For the CIO,

$\mathcal{N}_{\varepsilon}(\eta, \sigma)=f[\eta+\varepsilon \sigma]$,

$2 T(\eta, \sigma)=f[\eta+\sigma]+f[\eta-\sigma]$,

$2 R(\eta, \sigma)=f[\eta+\sigma]-f[\eta-\sigma]$.

Differentiation gives

$\boldsymbol{n}_{\varepsilon}=A^{\varepsilon}(\mathrm{d} \eta+\varepsilon \mathrm{d} \sigma)$, 
where I have defined $A^{\varepsilon}(\eta, \sigma):=A(\eta+\varepsilon \sigma)$. Sum and difference lead to

$\mathrm{d} T=\left(A^{+}+A^{-}\right) / 2 \mathrm{~d} \eta+\left(A^{+}-A^{-}\right) / 2 \mathrm{~d} \sigma$

and

$\mathrm{d} R=\left(A^{+}-A^{-}\right) / 2 \mathrm{~d} \eta+\left(A^{+}+A^{-}\right) / 2 \mathrm{~d} \sigma$,

and thus

$N^{2}(\eta, \sigma)=\frac{A(\eta)^{2}}{A^{+} A^{-}}$.

The parallel transport of the velocity of the CIO along the light rays leads to

$\tilde{\boldsymbol{U}}^{\varepsilon}=\frac{1}{A^{\varepsilon}}\left[\left(\left(A^{\varepsilon}\right)^{2}+A^{2}\right) \mathrm{d} \eta+\varepsilon\left(\left(A^{\varepsilon}\right)^{2}-A^{2}\right) \mathrm{d} \sigma\right]$.

\subsection{Space for the inertial cosmological observer}

Space, i.e., the surface $\Sigma_{\tau}$, has the equation

$f[\eta+\sigma]+f[\eta-\sigma]=2 \tau$, with $\sigma<\eta$,

which is not the surface $\eta=C^{\text {te }}$ (except in the case with no expansion, where $\eta=\tau$ ). In other worlds, space for the CIO is not a spatial section with maximal symmetry: the cosmic expansion breaks the spatial homogeneity (although not its isotropy when the observer is inertial). The spatial sections $\eta=C^{\text {te }}$, sometimes quoted as "space" do not verify the synchronicity condition (they verify a kind of synchronicity condition, but in the conformal time which has no physical relevance for the observer, rather than in its proper time).

Also, the cosmic expansion imprints a curvature onto space: even when space-time admits spatial sections of constant curvature (like for instance flat in the Einsteinde Sitter case), this is not the case for the space. Space is limited by the horizon $\sigma=\eta$, or $T=R$. On the horizon, $A^{-} \rightarrow 0$ and thus $N \rightarrow \infty$. Space tends to become null (light-like).

The $P T$-interval to a point of this surface is

$R=f[\eta+\sigma] / 2-f[\eta-\sigma] / 2=f[\eta+\sigma]-T$.

\section{The Einstein-de Sitter model}

To check this in more detail, I consider the example of Einstein-de Sitter space-time, with $A(\tau)=\tau^{2 / 3}=(\eta / 3)^{2}$ and flat spatial sections. In this case, $\eta(\tau)=3 \tau^{1 / 3}$ and $f(y)=(y / 3)^{3}$. Thus, $\Sigma_{\tau}$ has the equation (Fig. 6$)$

$27 \tau=\eta\left(\eta^{2}+3 \sigma^{2}\right)$,

and not $\tau=\eta^{3} / 27$, the equation of a flat spatial section. We have $N(\eta, \sigma)=\frac{\eta^{2}}{\eta^{2}-\sigma^{2}}=\left[1+{ }^{3} \sqrt{\frac{\tau+R}{\tau-R}}+{ }^{3} \sqrt{\frac{\tau-R}{\tau+R}}\right] / 4$. The $P T$-interval to a point $x$ is $R=\frac{\sigma}{27}\left(3 \eta^{2}+\sigma^{2}\right)$, different from the usual proper distance at time $\eta$, namely $\sigma A(\eta)=$ $\sigma \eta^{2} / 9$. Space, at the position of the observer, is orthogonal to its world line, as expected.

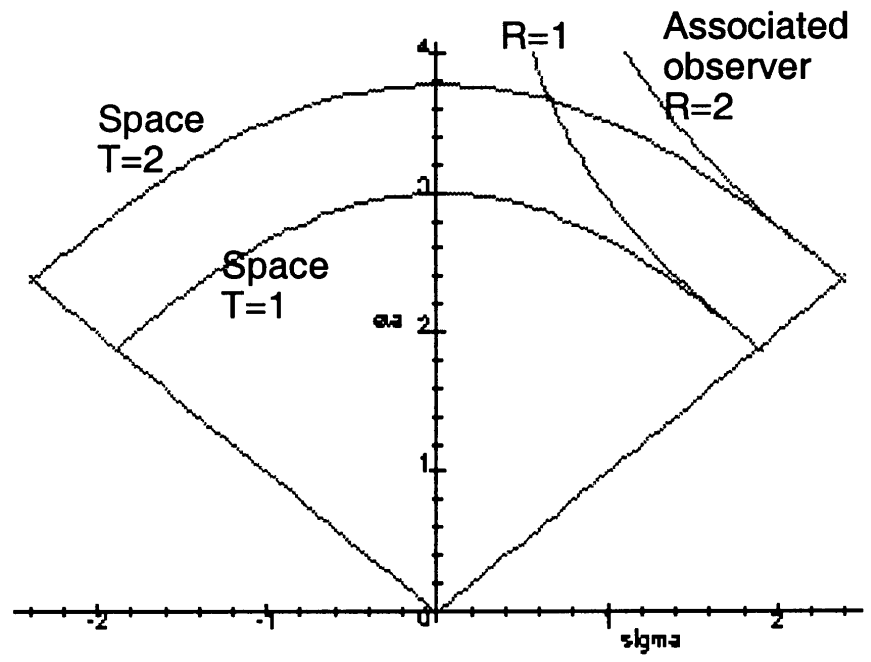

Fig. 6. Space ( $T=$ const.) and world line of associated observers $(R=$ const.) for the CIO in Einstein-de Sitter spacetime.

\section{Associated observers}

As we have seen, any observer has a class of associated observers. We point our that these observers are not the comoving observers defined by $\sigma=$ const., which obey the equation $\mathrm{d} T=\frac{A^{+}+A^{-}}{A^{+}-A^{-}} \mathrm{d} R$. On the other hand, the associated observers keep a constant PT-interval and obey the equation

$\mathrm{d} R=\left(A^{+}-A^{-}\right) / 2 \mathrm{~d} \eta+\left(A^{+}+A^{-}\right) / 2 \mathrm{~d}|\sigma|=0$.

An associated observer at the horizon is seen by the CIO with a redshift $z^{+} \rightarrow \infty$.

\section{Time and distances}

All measurements made by an observer, local or not, refer to his proper time. Thus, when a CIO considers an event in space-time, the relevant time to measure durations, or to date the event, is not $t$ or $\eta$ but $T$ defined above (I recall that $T$ and $t$ coincide on the world line of the CIO).

On the other hand, the proper distance is intended to measure the interval between two objects considered simultaneously, i.e., at a common value of time. But, again, no observer has access to the conformal time $\eta$. Thus, simultaneity (not absolute, but only relative to the observer) must be defined not by $\eta$ but by $T$ as we have explained. This leads to use the proper time distance (that I call differently to avoid confusion) between two objects, calculated by integration of the metric element, not along a spatial section $t=C^{\text {te }}\left(\right.$ or $\left.\eta=C^{\text {te }}\right)$, but along $\Sigma_{T}$, i.e.,

$\mathrm{d}_{P T}(g)=\int_{\Sigma_{T}} \mathrm{~d} s=\int_{\Sigma_{T}} N \mathrm{~d} R$

The PT-distance is thus really the distance between two objects in space, at a given moment for the CIO. 
Thus, for the CIO, $T$ and $R$ appear as convenient coordinates to measure space and time.

\subsection{Inertial observer in de Sitter space-time}

The case of the de Sitter space-time is particularly interesting since, because of its maximal symmetry it has been widely considered as a frame for quantization. As it is well known, it admits different slicings, with spatial sections of constant curvature with positive, zero or negative signs. To each slicing corresponds a convenient system of coordinates. Here I chose the slicing where spatial sections have constant positive curvature since it covers the whole space-time (de Sitter hyperboloid).

The metric is written as

$$
\begin{aligned}
\mathrm{d} s^{2} & =\mathrm{d} t^{2}-\rho^{2}\left(\cosh \rho^{-1} t\right)^{2}\left[\mathrm{~d} \sigma^{2}+\sin ^{2} \sigma \mathrm{d} \Omega^{2}\right] \\
& =A^{2}(\eta)\left[\mathrm{d} \eta^{2}-\mathrm{d} \sigma^{2}-\sin ^{2} \sigma \mathrm{d} \Omega^{2}\right]
\end{aligned}
$$

where the conformal time $\eta=\rho \tan ^{-1}\left[\sinh \left(\rho^{-1} t\right)\right]$ and $A(\eta)=\rho / \cos (\eta / \rho)$ ( $\rho$ is a constant characterizing the constant negative curvature of space-time).

The chosen CIO has proper time $t=$ $\rho \sinh ^{-1}[\tan (\eta / \rho)]$ so that $f(y):=\rho \sinh ^{-1}[\tan (y / \rho)]$. Thus,

$\mathcal{N}_{\varepsilon}(t, \sigma)=\rho \ln \frac{1+S^{\varepsilon}}{C^{\varepsilon}}$,

where $S^{\varepsilon}:=\sin \frac{\eta+\varepsilon|\sigma|}{\rho}, C^{\varepsilon}:=\cos \frac{\eta+\varepsilon|\sigma|}{\rho}$.

We obtain

$2 T(\eta, \sigma)=\rho \ln \frac{\cos (\sigma / \rho)+\sin (\eta / \rho)}{\cos (\sigma / \rho)-\sin (\eta / \rho)}$,

$2 R(\eta, \sigma)=\rho \ln \frac{\cos (\eta / \rho)+\sin (\sigma / \rho)}{\cos (\eta / \rho)-\sin (\sigma / \rho)}$.

Space for the CIO at (proper) time $\tau$ is given by

$\cos (\sigma / \rho)+\sin (\eta / \rho)=\exp (2 \tau / \rho)[\cos (\sigma / \rho)-\sin (\eta / \rho)],(60)$

or

$\cos (\sigma / \rho) \tanh (\tau / \rho)=\sin \left(\rho^{-1} t\right)$

which, again, is not the surface $t=$ const. of constant (positive) curvature. Thus we claim that quantization must be performed with this surface and its orthogonal time. This will be studied in a forthcoming paper.

\section{Discussion}

The prescription based on synchronicity defines space without ambiguity for any given observer, inertial or not, in arbitrary space-time (without multi-crossing of null geodesics), including Minkowski spacetime and the Friedmann-Lemaître models. Space is relative to the observer (it is in general different for an other observer), and well defined at each instant of its world line. This provides a foliation of space-time, valid for this observer, which may be interpreted as a class of canonically associated observers or a "kinematics" of space-time (Smarr \& York 1978). This provides also a natural reference frame, i.e., global space and time coordinates in the whole spacetime, which remains Minkowskian along the world line of the observer (thus, time coincides with its proper time there) and which is pertinent for physical measurements. In many cases (in particular for Rindler observers; see all references concerning the Rindler effect, and Sriramkumar \& Padmanabhan 1999), the coordinate system introduced here coincides with that used in the literature with no other justification than being "natural", and thus provides an a posteriori justification. Also, the prescription presented here appplies to a range wider than other reference frames.

Application to Minkowski spacetime confirms that space and time differ for inertial observers with different velocities. It provides an unambiguous and global definition of space and time for the Langevin observer, for which the other prescriptions do not apply. Applied to the Rindler observer (with uniform acceleration), it leads to space and time coordinates which coincide with the usual Rindler coordinates, thus providing a justification of their use. The corresponding interpretation of the Unruh effect involves the observer-dependant character of space and time.

In cosmology, this prescription provides, for the inertial observer in the general Friedmann-Lemaitre model, an unambiguous definition of space, which does not coincide with a spatial section of maximal symmetry. Thus, in the Friedmann-Lemaître models, no inertial observer "sees" a homogeneous space. The lack of homogeneity of space is due to the curvature corresponding to the expansion law. In particular, space is not flat or homogeneous (although the inertial character of the observer preserves its isotropy) in the Einstein-de Sitter model, sometimes called a "flat universe"! I have also calculated space for the inertial observer in de Sitter space-time, which, again, is not a hypersurface of maximal symmetry.

These results do not modify the cosmological formulae when they are expressed in a covariant form and do not involve a definition of space. However they change those interpretations of observational results, which involve a reference to space (like "space is homogeneous flat" etc.). This modifies also the interpretation of the usual proper distance: it does not appear as the proper spatial interval between two events occurring at the same time, but rather as a mixed interval between two events which are not synchronous for the observer which performs the measurement (they would be synchronous if the observer were using a watch indicating "conformal time" rather than "proper time"). Thus I introduce a "proper timedistance", which represents a spatial interval between two events which are synchronous for the observer. It corresponds to the result of a practical measurement that the observer may perform with his watch indicating his proper time. Its value differs, in general, form the usual proper distance. 
This prescription for space could have important implications for interpreting quantum effects in curved spacetime, and/or from the point of view of non-inertial observers. Its application to the Rindler observer confirms the usual results of the Unruh effect, and allows a clearer comprehension. In other cases, the prescription adopted here differs from most attempts up to now, since the use of spatial sections with maximal symmetry (rather than space as defined here) does not obey the synchronicity requirements. This suggest a new examination of quantum effects in curved space-time, to be discussed in a forthcoming paper. Also, subsequent work will explore in more detail the application of this procedure to observers with arbitrary acceleration and rotation.

\section{References}

Ali, S. T., Antoine, J.-P., \& Gazeau, J.-P. 1990, Ann. Inst. Henri Poincaré, 52, 83

Bel, L. 1998 [gr-qc/9812062]

Birrel, N. D., \& Davis, P. C. W. 1982, Quantum fields in curved space (Cambridge University Press)

Dolby, C. E., \& Gully, S. F. 2001 [gr-qc/0104077]

Landau, L., \& Lifshitz, E. 1966, Field Theory, MIR (URSS)

Marzlin, K.-P. 1994 [gr-qc/9402010 v2]

Misner, C. W., Thorne, K. S., \& Wheeler, J. A. 1973, Gravitation (Freeman and co.)

Pauri, M., Vallisneri, M. 1999 [gr-qc/9903052 v2]

Smarr, L., \& York, J. W. Jr. 1978, Phys. Rev. D, 17(10), 2329

Sriramkumar, L., \& Padmanabhan T. 1999

[gr-qc/9903054 v2] 